\title{
Stroke severity, early recovery and outcome are each related with clinical classification of stroke: data from the Tinzaparin in Acute Ischaemic Stroke Trial' (TAIST)
}

Nikola Sprigg MRCP; ${ }^{1}$ Laura J Gray MSC; ${ }^{1}$ Philip MW Bath MD $;{ }^{1}$ Ewa Lindenstrøm, MD; ${ }^{2}$ Gudrun Boysen, MD; ${ }^{3}$ Peter Paul De Deyn, MD ${ }^{4}$ Pal Friis, MD; ${ }^{5}$ Didier Leys, MD; ${ }^{6}$ Reijo Marttila, MD $;{ }^{7}$ Jan-Edwin Olsson, MD; ${ }^{8}$ Desmond O'Neill, FRCPI; ${ }^{9}$ Erich Bernd Ringelstein, MD; ${ }^{10}$ Jan-Jacob van der Sande, MD; ${ }^{11}$ Alexander GG Turpie, FRCP; ${ }^{12}$ for the TAIST Investigators

Institute of Neuroscience, ${ }^{1}$ University of Nottingham, Nottingham, UK; Leo Pharma $\mathrm{A} / \mathrm{S},{ }^{2}$ Ballerup, Denmark; Department of Neurology, ${ }^{3}$ Bispebjerg Hospital, Copenhagen, Denmark; Department of Neurology, ${ }^{4}$ A. Z. Middelheim, ZNA, University of Antwerp, Antwerpen, Belgium; Vest-Agder Sentralsykehus, ${ }^{5}$ Kristiansand, Norway; Clinique Neurologique, ${ }^{6}$ CHRU de Lille, Lille, France; Department of Neurology, ${ }^{7}$ Turku University Central Hospital, Turku, Finland; Institutionen för Neurologi, ${ }^{8}$ Universitetssjukhuset, Linköping, Sweden; Department of Age Related Health Care, ${ }^{9}$ Adelaide \& Meath Hospital, Dublin, Ireland; Klinik für Neurologie, ${ }^{10}$ Universität Münster, Münster, Germany; Slotervaartziekenhuis, ${ }^{11}$ Amsterdam, The Netherlands; Hamilton General Hospital, ${ }^{12}$ Hamilton, Canada

Corresponding author:

Professor Philip Bath

Division of Stroke Medicine

University of Nottingham

D Floor, South Block

Queen's Medical Centre

Nottingham NG7 2UH UK

Tel: 01158231023

Fax: 01158231022

Email: philip.bath@nottingham.ac.uk

Keywords: acute stroke; ischaemic stroke; recovery; functional outcome; clinical classification 


\section{ACKNOWLEDGMENTS AND FUNDING}

We thank Leo Pharma A/S for sharing the TAIST database. The analyses and their interpretation were performed independently of Leo Pharma A/S. LJG and NS are supported, in part, by The Stroke Association (UK) and BUPA Foundation (UK). PMWB is Stroke Association Professor of Stroke Medicine. 


\section{ABSTRACT}

Introduction: Baseline severity and clinical stroke syndrome (Oxford Community Stroke Project, OCSP) classification are predictors of outcome in stroke. We used data from the 'Tinzaparin in Acute Ischaemic Stroke Trial' (TAIST) to assess the relationship between stroke severity, early recovery, outcome and OCSP syndrome.

Methods: TAIST was a randomised controlled trial assessing the safety and efficacy of tinzaparin versus aspirin in 1,484 patients with acute ischaemic stroke. Severity was measured as the Scandinavian Neurological Stroke Scale (SNSS) at baseline and days 4, 7 and 10, and baseline OCSP clinical classification recorded: total anterior circulation infarct (TACI), partial anterior circulation infarct (PACI), lacunar infarct (LACI) and posterior circulation infarction (POCI). Recovery was calculated as change in SNSS from baseline at day 4 and 10. The relationship between stroke syndrome and SNSS at days 4 and 10, and outcome (modified Rankin scale at 90 days) were assessed.

Results: Stroke severity was significantly different between TACI (most severe) and LACI (mildest) at all four time points $(p<0.001)$, with no difference between PACI and POCI. The largest change in SNSS score occurred between baseline and day 4; improvement was least in TACI (median 2 units), compared to other groups (median 3 units) $(p<0.001)$. If SNSS did not improve by day 4 , then early recovery and late functional outcome tended to be limited irrespective of clinical syndrome (SNSS, baseline: 31 , day 10: 32; mRS, day 90: 4); patients who recovered early tended to continue to improve and had better functional outcome irrespective of syndrome (SNSS, baseline: 35, day 10: 50; mRS, day 90: 2).

Conclusions: Although functional outcome is related to baseline clinical syndrome (best with LACI, worst with TACI), patients who improve early have a more favourable functional outcome, irrespective of their OCSP syndrome. Hence, patients with a TACI syndrome may still achieve a reasonable outcome if early recovery occurs. 


\section{INTRODUCTION}

Baseline stroke severity, as assessed using measures such as Scandinavian Neurological Stroke Scale (SNSS) $)^{1,2}$ or National Institute Health Stroke Scale (NIHSS), ${ }^{3}{ }^{4}$ is widely accepted as the strongest clinical predictor of outcome in stroke; severe stroke is associated with an increased risk of death, disability and length of hospital stay, and institutionalisation. 3, 5-8 9 Similarly, severity, is also related to the early clinical course, with patients with severe stroke more likely to experience deterioration. ${ }^{10}$ In addition, recovery is observed later in severe stroke ${ }^{11}$ and it has been postulated that the extent of intrinsic cerebral damage is reflected not only by the degree of impairment, but also in the length of time to improvement after stroke. ${ }^{12}$

The importance of clinical history and examination, for evaluating stroke patients is well recognised, ${ }^{13}$ and stroke can be classified into four clinical syndromes, which have distinct natural histories: total anterior circulation syndrome (TACS), partial anterior circulation syndrome (PACS), lacunar syndrome (LACS) and posterior circulation syndrome (POCS). TACS is typically associated with greatest severity and worse outcome, PACS the highest risk of recurrence, whilst LACS has the mildest severity, and POCS the most favourable outcome. ${ }^{14}$ Clinical classification is also predictive of the risk of deterioration, with TACS having the greatest risk and LACS the least; deterioration is associated with a worse prognosis. ${ }^{15}$

The relationship between clinical classification and degree and timing of stroke recovery is less clear, and has been further examined here using data from the 'Tinzaparin in Acute Ischaemic Stroke Trial' (TAIST). ${ }^{16}$ 


\section{METHODS}

\section{Subjects}

TAIST compared the safety and efficacy of tinzaparin (low molecular weight heparin) given at high dose (175 anti-Xa IU/kg/day), tinzaparin at medium dose (100 anti-Xa $\mathrm{IU} / \mathrm{kg} / \mathrm{day}$ ), and aspirin (300 mg od), in patients with acute ischaemic stroke.

Subjects were included within 48 hours of stroke onset. Non-trial anti-platelets or anti-coagulants could not be given during the treatment period (10 days). Management of hypertension and other factors such as carotid stenosis was at the discretion of the local physician. All data were collected prospectively as part of the trial protocol.

\section{Clinical stroke classification}

Investigators prospectively recorded baseline clinical stroke syndrome as part of the trial protocol, and used the Oxford Community Stroke Project (OCSP) classification. ${ }^{14}$ All patients had haemorrhagic stroke excluded on the basis of computed topographic (CT) head scan and therefore were classified as total anterior circulation infarct (TACI), partial anterior circulation infarct (PACI), lacunar infarct (LACI) and posterior circulation infarct (POCI).

\section{Recovery}

Severity/impairment was measured at four different time points: baseline, day 4, 7 and 10 using the Scandinavian Neurological Stroke Scale (SNSS), which ranges from 0 (most severe) to 58 (no deficit), with death recorded as -1 . The rate of recovery was calculated as the change in SNSS at each time point. For comparison, patients were split into two groups: those who improved early (improvement $\geq 3$ on SNSS between baseline and day 4); and those who did not; 3 points was chosen as this was the median change in SNSS at day 4 . Day 4 was chosen as the largest change in SNSS occurred between baseline and day 4 and those with day 4 SNSS score missing were not categorised..

\section{Outcomes}

Outcome was assessed using the modified Rankin Scale (mRS) and Barthel Index (BI) at day 90 and recorded by face-to-face interview. Discharge disposition was classified as patients own home or other (such as institution). 


\section{Events}

Adverse events were recorded prospectively as part of the trial protocol including neurological deterioration (ND, a decrease in SNSS $\geq 5$ points), recurrent stroke (RS, either ischaemic, haemorrhagic or unclassified), venous thromboembolism (deep vein thrombosis and/or pulmonary embolism), and extracranial bleeding. Other events, such as pneumonia, and cardiac events (myocardial syndrome, dysrhythmia) were also recorded. Event rates at day 4 were analysed pooled for clinical classification and then by early improvers and non improvers. Whilst some patients had more than one adverse event, the categories ND, RS and HTI were considered exclusive.

\section{Statistical methods}

Data are given as frequency (\%), mean (standard deviation) or median (interquartile range) as appropriate. Patient characteristics and prognostic factors were compared by OCSP subgroup, using Chi-square, Wilcoxon and t-tests. Area under the curve (AUC) was used to compare the patterns of recovery by group. Change in SNSS was calculated at each time point and compared using Kruskal-Wallis Test. The Wilcoxon test was used to compare outcome in those who improved early with those who did not within each OCSP group. All analyses were performed using SAS (SAS Inst., USA). Significance was taken at $p<0.05$ and $95 \%$ confidence intervals are given. 


\section{RESULTS}

\section{Subjects}

Of 1,499 randomised patients, emerging exclusion criteria prevented treatment in 15, with analyses performed on the remaining 1,484 patients (figure 1). The baseline characteristics of included patients are shown in table 1 . Similar numbers of patients had TACI and PACI, whilst few had a POCI. Those with POCI tended to be slightly younger, and TACI patients were older and more likely to be male. There was a higher frequency of atrial fibrillation in patients with TACI, whilst patients with LACI had increased frequency of diabetes, and baseline blood pressure was also the highest (table 1). Patients with TACI had the most severe stroke severity (median SNSS 22) and LACI the least (median SNSS 40).

\section{Recovery}

Stroke impairment differed significantly between TACI (most severe), PACI and POCI (intermediate) and LACI (mildest), at all four time points (mean AUC $p<0.001$ ) (figure 2a). There was no difference in impairment between PACI and POCI. The greatest change in SNSS score occurred between baseline and day 4 (median improvement 3), with the least change in TACI (median 2) compared to PACI, LACI and POCI (median 3) $(p<0.001)$ (table 2). Patients who had not improved by day 4 (i.e. SNSS change $<3$ ) only had a limited improvement thereafter (median, baseline: 31, day 10: 32). In contrast, those who improved by day 4 (SNSS increased by $\geq 3$ ) have a much greater improvement thereafter (median, baseline: 35, day 10: 50). A similar pattern was still seen when sub dividing improvers and non-improvers by clinical syndrome; notably patients with TACI who improve by day 4 still had a comparable day 10 SNSS score to those PACI, LACI or POCI who had not improved (figure 2b). After day 4, there was no difference between clinical subgroups in the rate of recovery observed.

\section{Outcome}

The modified Rankin Scale and Barthel Index differed significantly between TACI and the other stroke syndromes, in both improvers and non-improvers (table 3 ). Improvers in each clinical group had a significantly better outcome at day 90 compared to those who had not improved at day 4 . These differences persisted after adjustment for age. Notably, TACI improvers had similar outcomes to PACI, POCI and LACI, who had not improved (table 3 ). Improvers were significantly more likely to be discharged home $(81.5 \%)$ than non-improvers $(66.3 \%)(p<0.001)$. 


\section{Adverse Events}

Neurological deterioration (ND) and recurrent stroke (RS, ischaemic or haemorrhagic) events were confined to non-improvers at day 4, whilst other events (such as cardiac events and VTE) occurred in both improvers and non-improvers (table 4).

Deterioration occurred more frequently than recurrence; TACI patients had the highest frequency of both ND and RS in comparison to patients with LACI who had the lowest frequency (table 4). Similarly, patients with TACI had the highest rate of any adverse event whilst LACI had the lowest. 


\section{DISCUSSION}

In keeping with previous studies we have demonstrated that baseline severity varies between clinical stroke syndromes, being mildest in LACI and most severe in patients with TACI. That this difference in severity persists throughout the first 10 days post stroke has not been previously described. Perhaps unsurprisingly, the rate of recovery also varies between syndromes, again with LACI improving most and fastest and TACI least and slowest.

In the first ten days, most recovery occurred early (by day 4 ), following which time recovery was more linear. ${ }^{6,17}$ We therefore dichotomised patients into 'improvers' and 'non-improvers', dependent on their rate of recovery at day 4 , and the recovery patterns of these sub-groups were distinct from one another. Furthermore, early improvement was significantly associated with a good outcome. Spontaneous recovery and association with good outcome is well documented in stroke ${ }^{17-19}$ although in comparison to this group of patients, previous studies included either only moderate ${ }^{19}$ or very severe stroke. ${ }^{19}$ The relationship between early improvement and outcome, across the range of stroke severity studied here, in patients within each clinical syndrome has not previously been documented. Furthermore the changes seen here were modest (median change $\geq 3$ SNSS points) yet were still associated with a clinically significant improvement in outcome.

Unlike aetiological classification, which is dependent on the results of ancillary investigations, ${ }^{20}$ clinical classification is simple and can be performed with reasonable reliability on initial assessment of the patient. ${ }^{21}$ Similarly, the further assessment of impairment as a measure of recovery during the first week after stroke can strengthen ability to predict outcome. ${ }^{22}$ This makes the combination of clinical classification and monitoring of recovery an attractive tool, both clinically and for research where the ability to accurately predict recovery and outcome is key. This may be especially beneficial in subgroups of patients thought typically to have a poorer outcome, such as TACS, since there appears that there is at least one subgroup of patients who may do relatively well.

Potential reasons for non improvement were analysed and were insufficient to account for lack of improvement in the majority of patients. However, recovery mechanisms are poorly understood and may relate to angiological factors, including spontaneous lysis, thrombus migration and transcortical perfusion networks, ${ }^{23}$ which were not 
studied directly here. Beyond these vascular factors, recover may also be intrinsically linked to the brain's plastic potential, ${ }^{24}$ not withstanding that certain events may intervene and prevent recovery.

A caveat in our findings is that by assessing recovery in terms of severity (SNSS), multivariate analysis was not possible to ascertain if outcome was related to severity, as has been previously well documented. Furthermore, selection bias may have been present since we studied patients enrolled into the randomised trial; limited inclusion criteria will mean that some groups of patients were under represented (e.g. POCI) whilst the trial excluded patients with very minor or very severe strokes. Similarly, due to the long recruitment time window in TAIST (48 hours), stroke impairment will have changed in some patients prior to enrolment, and also patients who may have improved or deteriorated rapidly during the first hours will have been excluded. Future studies should focus on patients with hyperacute stroke. Despite this a large number of patients continue to present late to medical practitioners. Finally, limitations of clinical classification are well documented, ${ }^{21}$ with concordance for the diagnosis of PACS less than for that for TACS and LACS. ${ }^{25}$

Despite these points, TAIST contains high quality data on a large number of patients in a population where no treatment effect was observed, hence making it appropriate for hypothesis generating analyses. This study suggests that early recovery is related to clinical stroke syndrome, with LACI having the greatest recovery and most favourable outcome, and TACI having the least recovery and poorest outcome. However, significant recovery can occur in patients traditionally thought to have a poor prognosis, such as TACI and PACI, with clinically significant improvement in outcome.

These data, if confirmed, suggest that early monitoring of recovery using a simple clinical classification and impairment scale such as OCSP and SNSS respectively, may help in predicting prognosis and possibly deciding which patients to recruit into trials. 


\section{TABLE 1}

Baseline characteristics by stroke type. Number (\%), mean (standard deviation) or median (inter quartile range).

\begin{tabular}{|c|c|c|c|c|c|c|}
\hline & \multirow[t]{2}{*}{ Total } & \multicolumn{4}{|c|}{ OCSP Classification } & \multirow[t]{2}{*}{$P$ value } \\
\hline & & LACI & TACI & PACI & POCI & \\
\hline Subjects (\%) & 1484 & $422(28.4)$ & $523(35.2)$ & $473(31.9)$ & $66(4.5)$ & \\
\hline Age (years) & $71.6(11.0)$ & $70.7(11.1)$ & $72.6(11.0)$ & $71.7(10.6)$ & $68.5(11.2)$ & 0.006 \\
\hline Male (\%) & $807(54.4)$ & $233(55.2)$ & $283(54.1)$ & $249(52.6)$ & $42(63.6)$ & 0.39 \\
\hline SNSS (median (IQR)) & $34(24-42)$ & $40(33-47)$ & $22(12-30)$ & $37(29-45)$ & $40(30-46)$ & $<0.0001$ \\
\hline Systolic BP (mmHg) & $156.4(22.8)$ & $160.1(22.6)$ & $154.5(22.1)$ & $155.4(23.7)$ & $156.2(20.5)$ & 0.001 \\
\hline Diastolic BP (mmHg) & $84.6(12.1)$ & $86.0(12.1)$ & $84.3(12.3)$ & $83.9(12.0)$ & $83.5(11.3)$ & 0.05 \\
\hline Previous stroke (\%) & $192(13.2)$ & $52(12.4)$ & $66(12.8)$ & $67(14.5)$ & $8(12.1)$ & 0.82 \\
\hline Previous TIA (\%) & $241(16.3)$ & $71(16.9)$ & $73(14.7)$ & $86(18.2)$ & $11(16.7)$ & 0.46 \\
\hline Atrial fibrillation (\%) & $181(21.4)$ & $21(9.1)$ & $109(32.8)$ & $48(20.0)$ & $3(7.1)$ & $<0.0001$ \\
\hline Diabetes (\%) & $250(16.9)$ & $91(21.6)$ & $81(15.5)$ & $67(14.2)$ & $11(16.7)$ & 0.02 \\
\hline Previous MI (\%) & $232(15.6)$ & $62(14.7)$ & $90(17.6)$ & $70(14.8)$ & $10(15.2)$ & 0.62 \\
\hline
\end{tabular}

BP: blood pressure; MI: myocardial syndrome; SNSS: Scandinavian Neurological Stroke Scale; TIA: transient ischaemic attack 


\section{TABLE 2}

Rates of recovery by OCSP classification. Median (inter quartile range); comparison by the Kruskal-Wallis Test.

\begin{tabular}{|c|c|c|c|c|c|c|}
\hline & Total & LACI & TACI & PACI & POCI & $\mathrm{P}$ value \\
\hline Change baseline - Day 4 & $3(0-7)$ & $3(0-7)$ & $2(-2-6)$ & $3(0-7)$ & $3(-1-9)$ & 0.001 \\
\hline Change Day 4 - Day 7 & $2(0-4)$ & $2(0-4)$ & $2(0-4)$ & $2(0-4)$ & $0(0-4)$ & 0.06 \\
\hline Change Day 7 - Day 10 & $0(0-3)$ & $1(0-3)$ & $0(0-3)$ & $0(0-3)$ & $1(0-3)$ & 0.08 \\
\hline Change baseline - Day 10 & $6(2-12)$ & $7(3-11)$ & $6(0-12)$ & $7(2-12)$ & $6(0.5-12)$ & 0.08 \\
\hline
\end{tabular}




\section{TABLE 3}

Outcome at day 90 by stroke type and type of recovery. Median (inter quartile range); comparison by the Wilcoxon test.

\begin{tabular}{lccc}
\hline & Early improvers & Non improvers & P value \\
\hline Rankin Scale & $2(1-3)$ & $3(2-4)$ & $<0.0001$ \\
LACI & $3(2-4)$ & $4(4-6)$ & $<0.0001$ \\
TACI & $2(1-3)$ & $3(3-2)$ & $<0.0001$ \\
PACI & $2(1-3)$ & $3(2-5)$ & 0.002 \\
POCI & & & \\
Barthel Index & $95(85-100)$ & $85(60-95)$ & $<0.0001$ \\
LACI & $85(35-100)$ & $22(-5-65)$ & $<0.0001$ \\
TACI & $97.5(85-100)$ & $70(40-90)$ & $<0.0001$ \\
PACI & $100(87.5-100)$ & $60(0-95)$ & 0.001 \\
POCI & & & \\
\hline
\end{tabular}




\section{TABLE 4}

Adverse events at day 4 by improver/non improver status and clinical classification. Those with day 4 SNSS score missing were not categorised. Some patients had more than one event. Number (\%).

\begin{tabular}{|c|c|c|c|c|c|c|c|c|c|c|}
\hline & \multicolumn{5}{|c|}{ Improvers } & \multicolumn{5}{|c|}{ Non improvers } \\
\hline & Total & LACI & TACI & PACI & POCI & Total & LACI & TACI & PACI & POCI \\
\hline & 732 & 216 & 224 & 256 & 36 & 703 & 187 & 289 & 197 & 30 \\
\hline Neurological deterioration & & 0 & 0 & 0 & 0 & $130(18.5)$ & $20(10.7)$ & $71(24.6)$ & $3216.2)$ & $7(23.3)$ \\
\hline Extracranial bleeding & $27(3.7)$ & $8(3.7)$ & $11(4.9)$ & $6(2)$ & $2(5.6)$ & $36(5.1)$ & $11(5.9)$ & $12(4.1)$ & $9(4.6)$ & $4(13.3)$ \\
\hline VTE & $2(0.3)$ & 0 & $1(0.4)$ & $1(0.4)$ & 0 & $10(1.4)$ & $1(0.5)$ & $5(1.7)$ & $4(2.0)$ & 0 \\
\hline Recurrent stroke* & 0 & 0 & 0 & 0 & 0 & $31(4.4)$ & $4(2.1)$ & $15(5.2)$ & $11(5.6)$ & $1(3.3)$ \\
\hline Haemorrhagic stroke & 0 & 0 & 0 & 0 & 0 & $9(1.3)$ & 0 & $7(2.4)$ & $2(1.0)$ & 0 \\
\hline Pneumonia & 0 & 0 & 0 & 0 & 0 & $10(1.4)$ & $2(1.1)$ & $7(2.4)$ & $1(0.5)$ & 0 \\
\hline Cardiac events & $6(0.8)$ & 0 & $1(0.4)$ & $3(1.2)$ & $2(5.6)$ & $17(2.4)$ & $3(1.6)$ & $8(2.8)$ & $6(3.0)$ & 0 \\
\hline HTI & $2(0.3)$ & 0 & $1(0.4)$ & 0 & $1(2.7)$ & $1(0.1)$ & 0 & 0 & $1(0.5)$ & 0 \\
\hline Others & $4(0.5)$ & 0 & $2(0.9)$ & $2(0.8)$ & 0 & $11(1.7)$ & $3(1.6)$ & $6(2.1)$ & $2(1.0)$ & 0 \\
\hline Any event & $41(5.6)$ & $8(3.7)$ & $16(7.1)$ & $12(4.7)$ & $4(11.1)$ & $163(23.2)$ & $44(23.5)$ & $138(47.8)$ & $69(35.0)$ & $12(40.0)$ \\
\hline
\end{tabular}

*Ischaemic or unclassified, VTE venous thromboembolism, HTI haemorrhagic transformation of infarction 


\section{FIGURE 1}

Number of patients included in the analysis. Improvers have a change in SNSS between baseline and day 4 of greater than or equal to 3 points. Those with day 4 SNSS score missing were not categorised.






\section{FIGURE 2a}

Recovery by clinical stroke syndrome

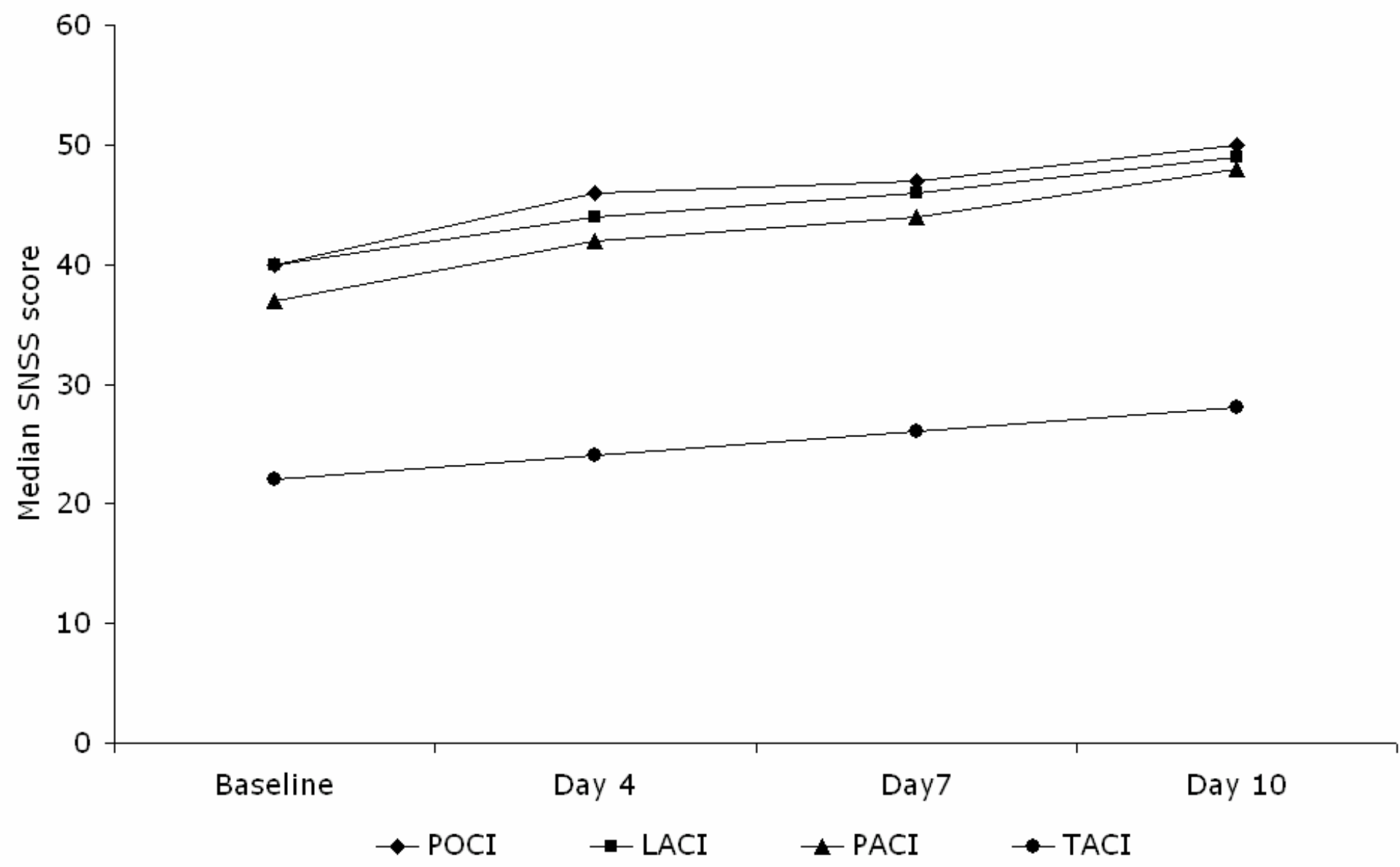




\section{FIGURE 2b}

SNSS at days $0,4,7,10$ by early improvement and stroke syndrome, broken lines indicate patients who had not improved to median of 3 SNSS points by day 4 .

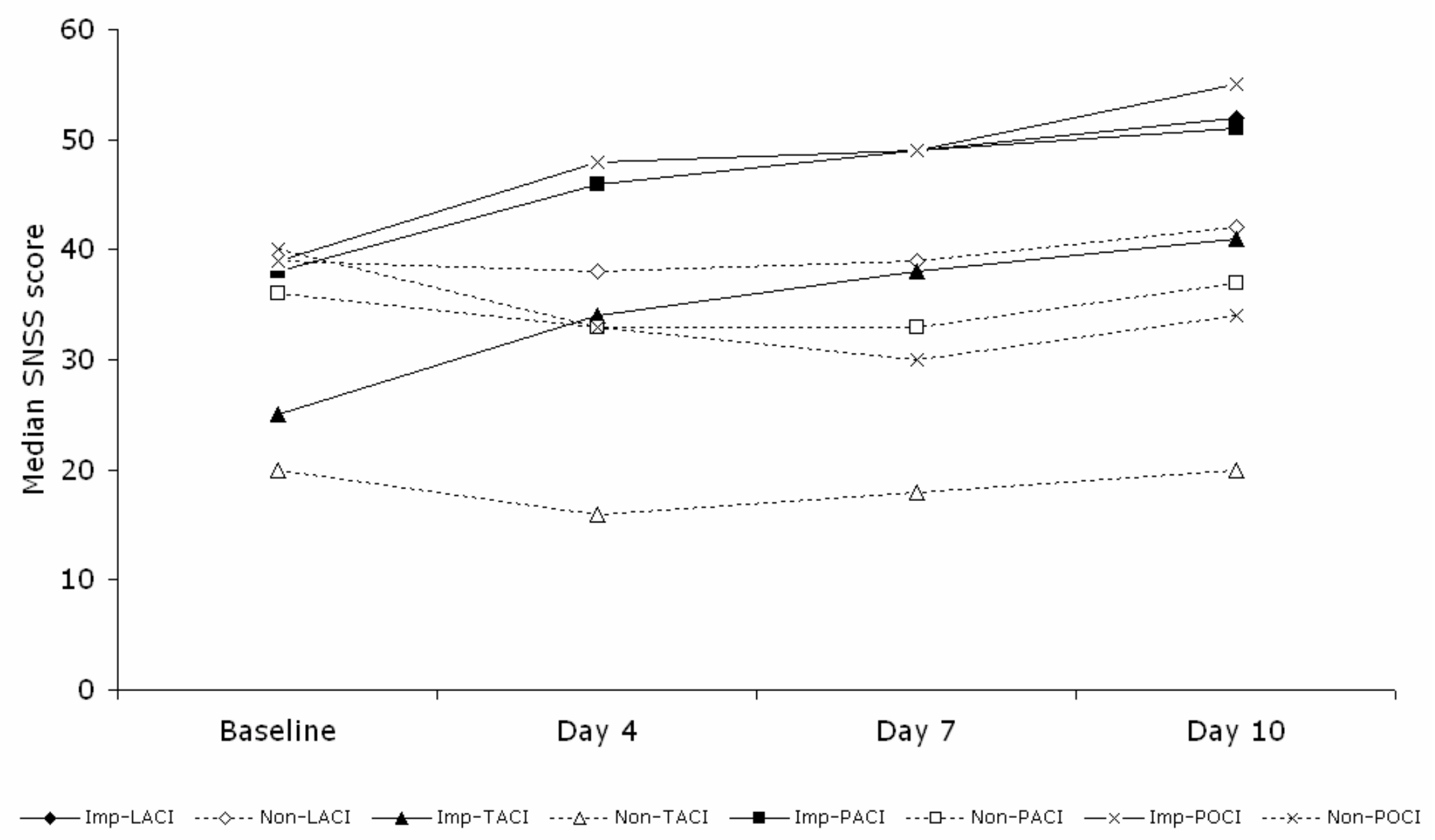




\section{References}

1. Scandinavian Stroke Study Group. Multicenter trial of hemodilution in ischemic stroke background and study protocol. Stroke. 1985;16:885-890

2. Lindenstrom E, Boysen G, Christiansen LW, Rogvi Hansen B, Nielsen PW, 1514. Reliability of Scandinavian neurological stroke scale. Cerebrovascular Diseases. 1991;1:103-107

3. Adams HP, Davis PH, Leira EC, Chang KC, Bendixen BH, Clarke WR, et al. Baseline NIH Stroke Scale score strongly predicts outcome after stroke. A report of the trial of Org 10172 in Acute Stroke Treatment (TOAST). Neurology. 1999;53:126-131

4. Brott T, Adams HP, Olinger CP, Marler JR, Barsan WG, Biller J, et al. Measurements of acute cerebral infarction: a clinical examination scale. Stroke. 1989;20:864-870

5. Reith J, Jorgensen S, Pedersen $P$, Nakayama H, Raaschou H, Jeppesen L, et al. Body temperature in acute stroke: relation to stroke severity, infarct size, mortality, and outcome. Lancet. 1996;347:422-425

6. Jorgensen HS, Nakayama H, Reith J, Raaschou HO, Olsen TS. Outcome and time course of recovery in stroke Part 1; Outcome. Copenhagen Stroke Study. Archives of Physical Medicine and Rehabilitation. 1995;76:406-412

7. Boysen G, Christemsen H. Stroke severity determines body temperature in acute stroke. Stroke. 2001;32:413-417

8. Christensen $\mathrm{H}$, Boysen G, Truelsen T. The Scandinavian Stroke scale predicts outcome in patients with mild ischemic stroke. Cerebrovascular Diseases. 2005;20:46-48

9. Jorgensen HS, Nakayama H, Raaschou HO, Olsen TS. Acute stroke: Prognosis and a prediction of the effect of medical treatment on outcome and health care utilization: The Copenhagen Stroke Study. Neurology. 1997;49:1335-1342

10. DeGraba TJ, Hallenbeck HM, Pettigrew KD, Dutka AJ, Kelly BJ. Progression in acute stroke: Value of the initial NIH stroke scale score on patient stratification in future trials. Stroke. 1999;30:1208-1212

11. Jorgensen HS, Nakayama H, Raaschou HO, Vive-Larsen J, Stoier M, Olsen TS. Outcome and Time Course of Recovery in Stroke. Part II: Time Course of Recovery. The Copenhagen Stroke Study. Archives of Physical Medicine and Rehabilitation. 1995;76

12. Kwakkel G, Kollen B, Lindeman E. Understanding the pattern of functional recovery after stroke: Facts and theories. Restorative Neurology and Neuroscience. 2004;22:281-289

13. Goldstein LR, L. SD. Is this patient having a stroke? Journal of American Medical Association. 2005;293:2391-2402

14. Bamford J, Sandercock P, Dennis M, Burn J, Warlow C. Classification and natural history of clinically identifiable subtypes of cerebral infarction. Lancet. 1991;337:1521-1526

15. Tei H, Uchiyama S, Ohara K, Kobayashi M, Uchiyama Y, Fukuzawa M. Deterioration Ischemic Stroke in 4 Clinical Categories Classified by the Oxfordshire Community Stroke Project. Stroke. 2000;31:2049-2054

16. Bath P, Lindenstrom E, Boysen G, De Deyn P, Friis P, Leys D, et al. Tinzaparin in acute ischaemic stroke (TAIST): a randomised aspirin-controlled trial. Lancet. 2001;358:702710

17. Jorgensen HS, Reith J, Nakayama H, Kammersgaard LP, Raaschou HO, Olsen TS. What determines good recovery in patients with the most severe strokes? The Copenhagen stroke study. Stroke. 1999;30:2008-2012

18. Biller J, Love BB, Marsh EE, Jones MP, Knepper LE, Jiang D, et al. Spontaneous improvement after acute ischemic stroke: a pilot study. Stroke. 1990;21:1008-1012

19. Wityk RJ, Pessin MJ, Kaplin RF, Caplan LR. Serial assessment of acute storke using the NIH stroke scale. Stroke. 1994;25:362-365

20. Madden KP, Karanjia PN, Adams HP, Clarke WR, and the TOAST Investigators. Accuracy of initial stroke subtype diagnosis in the TOAST study. Neurology. 1995;45:1975-1979

21. Lindley R, Warlow C, Wardlow J, Dennis M, Slattery J, Sandercock P. Interobserver reliability of a clinical classification of acute cerebral infarction. Stroke. 1993;24:18011804

22. Hendricks HT, van Limbeek J, Geurts AC, Zwarts MJ. Motor recovery after stroke: A systematic review of the literature. Archives of Physical Medicine and Rehabilitation. 2002;83:1629-1637 
23. Ringelstein EB, Biniek R, Weiller C, Ammeling B, Nolte PN, Thron MD, et al. Type and extent of hemispheric brain infarctions an clinical outcome in early and delayed middle cerebral artery recanlization. Neurology. 1992;42:289-298

24. Calautti C, Baron JC. Functional neuroimaging studies of motor recovery after stroke in adults: A review. Stroke. 2003;34:1553-1566

25. Aerden L, Luijckx GJ, Ricci S, Hilton A, Kessels F, Lodder J. Validation of the Oxfordshire Community Stroke Classification Project syndrome diagnosis derived from a standard symptom list in acute stroke. Journal of the Neurological Sciences. 2004;220:55-58 